\title{
APLICAÇÃO DA TÉCNICA REGRESSÃO LINEAR MÚLTIPLA: UM ESTUDO DE CASO EM UMA FÁBRICA DE CIMENTOS
}

\author{
APPLICATION OF THE MULTIPLE LINEAR REGRESSION TECHNIQUE: A CASE STUDY IN A
}

CEMENT FACTORY

Guilherme Maranhão Barreto Pereira ${ }^{1}$, Lorena Bittencourt Bastos ${ }^{2}$, Karine Gonçalves ${ }^{3}$, Gabriela da Rosa Witeck ${ }^{4}$, Edson Pacheco Paladini ${ }^{5}$

DOI: 10.37702/REE2236-0158.v39p50-60.2020

\section{RESUMO}

A busca constante pela excelência a fim de se alcançar a estabilidade operacional e de atender aos requisitos dos clientes tem sido um dos principais direcionadores dos esforços das empresas em um mercado cada vez mais competitivo e em um ambiente em que os clientes se mostram cada vez mais exigentes. A velocidade de resposta às inúmeras variações encontradas no processo de fabricação do cimento é um grande diferencial em um processo no qual o principal indicador de qualidade do produto é explorado 24 horas após sua fabricação. No entanto, é necessário conhecer como os parâmetros de processo se relacionam e influenciam na resistência final do cimento, de modo a se obter uma previsibilidade sobre o resultado, auxiliando a tomada de decisão na busca dos objetivos da qualidade. Na pesquisa utilizou-se uma metodologia descritiva do tipo quantitativa, com uma coleta de amostras de cimento que tiveram as seguintes características analisadas: índice de finura, blaine, índice de SO3 e índice de resíduo insolúvel. O presente artigo tem como objetivo avaliar as influências de quatro variáveis de processo no resultado de resistência à compressão em um dia de produção na fabricação de cimento, aplicando-se a técnica de regressão linear múltipla e, assim, demonstrar a equação que descreve a relação entre as variáveis independentes e a variável dependente.

Palavras-chave: análise de dados; regressão linear; correlação entre variáveis.

\begin{abstract}
The constant pursuit of excellence in achieving operational stability and meeting customer requirements has been a key factor of companies efforts in an increasingly competitive marketplace in an environment where customers are critical. The response speed of variations incident in the cement manufacturing process is a major differential, in which the main indicator of quality product is explored after twenty-four hours after manufactured. However, it is necessary to know how the process parameters are related to, and the influence in the final strength of the cement, in order to obtain a predictability about the result, helping decision making in pursuit of quality objectives. In the research, a quantitative descriptive methodology was used, with a collection of cement samples analyzing the following characteristics: fineness index, blaine, SO3, index and insoluble residue index. The purpose of this paper is to evaluate the influence of four process variables on the result of compressive strength, in one day of production in the cement manufacturing, applying the multiple
\end{abstract}

1 Pós-graduado em Engenharia de Produção Enxuta, PUC/PR. gmbp1983@gmail.com

2 Mestranda em Engenharia de Produção, Universidade Federal de Santa Catarina. lorenabbastos@yahoo.com.br

3 Pós-graduada em Engenharia de Produção Enxuta, PUC/PR. goncalveskarine79@gmail.com

4 Doutoranda em Engenharia Industrial, Universidade de Minho. gabiwiteck@gmail.com

5 Prof. Dr. em Engenharia de Produção, Universidade Federal de Santa Catarina. edson.paladini@ufsc.br 
linear regression technique and thus, to demonstrate the equation that describes the relation between the independent and dependent variables.

Keywords: data analysis; linear regression; correlation between variables.

\section{INTRODUÇÃO}

A indústria brasileira de cimento desempenha um papel importante no cenário nacional e internacional, ocupando a quarta posição entre os maiores produtores mundiais (ABCP, 2015). A busca constante pela excelência nos processos produtivos tem sido um dos principais direcionadores dos esforços das empresas em um mercado cada vez mais competitivo. De acordo com Paladini (2011), o contexto mercadológico, em que atuam as organizações, sofre alterações conceituais contínuas. Tal dinamismo requer novas formas de entender, viabilizar e gerenciar de acordo com as variações de elementos internos e cenários externos às organizações.

A velocidade de resposta às variações internas do processo de fabricação do cimento é um grande diferencial, uma vez que o principal indicador de qualidade do produto é evidenciado após vinte e quatro horas da sua fabricação. Para tanto, é necessário conhecer como os parâmetros de processo se relacionam e influenciam na resistência final do cimento, de modo a se obter uma previsibilidade sobre o resultado, auxiliando a tomada de decisão na busca dos objetivos da qualidade.

Neste contexto, em que os processos de fabricação apresentam variáveis que estão relacionadas entre si, a análise de regressão linear ou não linear permite investigar essa relação e desenvolver um modelo matemático conhecido como equação de regressão (HINES, 2006).

Este artigo visa ao desenvolvimento de um estudo de caso em uma organização cimenteira brasileira. Ademais, a pesquisa possui como objetivo aplicar técnicas de regressão linear múltipla, a fim de avaliar quais são as influências e variáveis de processo que poderão exercer impacto no resultado da resistência à compressão do produto fabricado.

\section{REFERENCIAL TEÓRICO}

Nas últimas décadas os pacotes computacionais estatísticos foram aperfeiçoados e o acesso foi facilitado para o usuário, resultando em um aumento na aplicação e produção científica com emprego de diversas análises para tratamento quantitativo dos dados (GOUVÊA et al., 2012).

Em muitos problemas, quando há duas ou mais variáveis que são intrinsecamente relacionadas, é necessário explorar a natureza dessa relação. A análise de regressão é uma técnica estatística para a modelagem e a investigação de relações entre duas ou mais variáveis, a relação entre essas variáveis é caracterizada por um modelo matemático chamado de equação de regressão (HINES, 2006).

Já a análise simultânea, com mais do que duas variáveis, é considerada uma análise multivariada, em que todas as variáveis são aleatórias e inter-relacionadas de maneira que seus diferentes efeitos não podem ser significativamente interpretados separadamente (HAIR JR. et al., 2009). Essa técnica de análise de dados multivariada permite o tratamento de diversas variáveis ao mesmo tempo, oferecendo ao pesquisador um material bastante robusto para a análise dos dados de pesquisa (GOUVÊA et al., 2012).

Hair Jr. et al. (2009) concluem que a técnica estatística de análise de dados denominada regressão simples baseia-se na análise que envolve apenas uma variável independente (X), enquanto a regressão múltipla é uma técnica estatística multivariada usada para examinar uma única variável dependente (Y) e um conjunto de variáveis 
independentes $\quad(\mathrm{X} 1+\mathrm{X} 2+\ldots .+\mathrm{Xn}), \quad$ sua formulação básica é definida por:

$$
\mathrm{Y} 1=\mathrm{X} 1+\mathrm{X} 2+\ldots .+\mathrm{Xn}
$$

Hair Jr. et al. (2009) ainda destacam que o pesquisador deve expandir o modelo de regressão simples acrescentando variáveis independentes para melhorar a capacidade de predição, descobrindo quais variáveis são mais importantes e quais podem ser ignoradas na análise. Gallo (2015) acrescenta que a análise de regressão é uma maneira de resolver matematicamente quais variáveis independentes realmente têm impacto sobre a variável dependente. Embora existam riscos em se tentar incluir muitas variáveis em uma análise de regressão, o impacto de múltiplas variáveis de uma só vez é uma das maiores vantagens da regressão.

Para aplicar a análise de regressão múltipla o pesquisador deve especificar os objetivos da análise, os dados utilizados devem ser métricos ou adequadamente transformados. Além disso, é necessário classificar as variáveis em dependentes - variável resposta - e independentes (HAIR JR. et al., 2009).

Com relação ao resultado da regressão, Gallo (2015) destaca que as variáveis correlacionadas em uma análise de regressão não provam a existência de uma relação de causa e efeito, ou seja, cabe ao pesquisador (analista) verificar o que ocorre de fato, pois correlação não é causalidade.

\section{PROCEDIMENTOS METODOLÓGICOS}

Esta seção tem como objetivo apresentar os procedimentos metodológicos utilizados para a realização deste trabalho. Assim, a seção está dividida em cinco etapas: i) classificação da pesquisa; ii) justificativa da técnica analisada; iii) contextualização do processo produtivo analisado; iv) coleta de dados; e v) análise de dados.

\section{Classificação da pesquisa}

O método de pesquisa utilizado no presente artigo é classificado como descritivo, apoiandose em coletas de dados quantitativas. De acordo com Minayo e Gomes (2012), a metodologia é a direção do pensamento, juntamente com a prática executada da realidade.

Segundo Andrade (2010), a pesquisa descritiva expõe fatos; estes são observados, registrados, analisados e interpretados, de modo que o pesquisador não interfira nos resultados da pesquisa.

Conforme Gil (2010), a pesquisa descritiva tem por finalidade descrever as características de uma dada população, de um fenômeno específico ou da correlação entre variáveis, utilizando-se técnicas para a coleta de dados, como aplicação de questionários e observações.

Ainda, de acordo com Michel (2015), a pesquisa quantitativa é compreendida através das informações, opiniões e problemas, expressados exclusivamente no formato de números. $\mathrm{O}$ autor relata que a pesquisa quantitativa é apresentada em coleta de dados que utilizam técnicas de estatísticas que variam das mais simples até as mais complexas.

Para Perovano (2014), a pesquisa descritiva constitui-se com base nas análises das relações entre variáveis, proporcionando especificações dos efeitos elaborados em determinados métodos por intermédio de análise e identificação de dados.

\section{Justificativa da técnica utilizada}

Como a variável dependente e as quatro variáveis independentes são contínuas, logo optou-se por utilizar a técnica de regressão linear múltipla. Essa decisão também se justifica ao se explorar e quantificar como estas variáveis independentes se relacionam e em que intensidade influenciam a variável dependente dentro do modelo apresentado. Portanto, a análise de regressão linear também associa outros procedimentos de diagnóstico, como a análise de resíduos e o teste de independência de Durbin-Watson, por exemplo, que indica a consistência do modelo e proporciona a visibilidade de como melhorá-lo. 


\section{Contextualização do processo produtivo analisado}

O objeto de estudo foi uma fábrica de cimentos, localizada na região Sul do país. A planta teve o seu startup em 2011 e possui capacidade instalada de produção anual de um milhão de toneladas de cimento. A produção de cimento é um processo contínuo com a combinação dos insumos dentro de um moinho tubular de bolas. Amostras do cimento produzido são coletadas a cada duas horas para ensaios de controle de processo: índice de finura, blaine, índice de SO3 e índice de resíduo insolúvel. Os ajustes no processo são realizados com base nos resultados obtidos nesses ensaios.

A planta não possui um laboratório de ensaios físicos para realização dos testes de resistência à compressão. Ao final do dia é composta uma amostra padrão - com a média das amostras coletadas - que é enviada diariamente para a outra fábrica da companhia a fim de se realizar os ensaios. Esse processo (de elaboração da amostra padrão e seu transporte) pode levar até 48 horas, corroborando a justificativa deste estudo em busca da previsibilidade do resultado de resistência à compressão com base nos resultados dos parâmetros de processo encontrados nas amostras coletadas.

\section{COLETA DE DADOS}

A amostra foi coletada do sistema de qualidade da empresa em outubro de 2018 e compreende o período de $1^{\circ}$ de janeiro de 2017 a 31 de dezembro de 2017. As variáveis independentes do modelo são: índice de finura, blaine, índice de $\mathrm{SO} 3$ e índice de resíduo insolúvel. A variável dependente é o resultado de resistência à compressão de um dia de produção, após a moldagem do corpo de prova. Foram analisadas 284 amostras de cada variável (independentes e dependente). Todas as variáveis são quantitativas contínuas.

A aplicação da técnica de regressão linear múltipla está associada ao cumprimento de alguns pré-requisitos que serão comprovados nos resultados da regressão. Os pré-requisitos estão listados a seguir:

a) distribuição normal da variável dependente;

b) ausência de multicolinearidade, que significa que as variáveis dependentes não devem apresentar uma forte correlação;

c) independência dos resíduos. Se os erros estiverem correlacionados, a regressão de mínimos quadrados pode subestimar o erro padrão dos coeficientes;

d) distribuição normal dos resíduos;

e) homoscedacidade.

Os procedimentos adotados, portanto, têm início com uma análise descritiva da normalidade da amostra, através de um teste de hipótese e com o teste de ausência de multicolinearidade e de correlação, aplicados separadamente.

A seguir o modelo será testado para verificar se as variáveis dependentes são capazes de prever $\mathrm{o}$ valor da variável dependente. Para tanto, será verificada a significância estatística do modelo, observando-se o resultado da Análise de Variância (ANOVA) e o resultado do Coeficiente de Determinação $\left(\mathrm{R}^{2}\right)$.

A análise dos resíduos quadrados será realizada para verificar a normalidade da distribuição, assim como se é verificada a independência dos valores residuais por meio do teste de Durbin-Watson. Também será verificado se as variáveis apresentam homoscedacidade e, como último pré-requisito, se existe a presença de outliers nos resíduos.

Validado o teste, serão observados os coeficientes padronizados para se identificar a relação entre as variáveis independentes do modelo e a variável dependente e também com que intensidade essas variáveis influenciam no resultado do modelo. Será observado, na análise dos coeficientes, quais coeficientes podem ser descartados do modelo através do teste de hipótese.

Por fim, será apresentada qual equação descreve a relação entre as variáveis 
independentes (X1, X2, X3 e X4) e a variável dependente $(\mathrm{Y})$.

\section{ANÁLISE DE DADOS}

Nesta seção será apresentada a análise de dados, de acordo com a coleta de dados obtidos na pesquisa e com a utilização do programa SPSS para a compilação e análise dos dados.

\section{Verificação da normalidade da distribuição da variável dependente}

Figura 1 - Teste de normalidade

\begin{tabular}{|c|c|c|c|c|c|c|}
\hline \multicolumn{7}{|c|}{ Testes de Normalidade } \\
\hline & \multicolumn{3}{|c|}{ Kolmogorov-Smirnov ${ }^{a}$} & \multicolumn{3}{|c|}{ Shapiro-Wilk } \\
\hline & Estatística & $g \mid$ & Sig. & Estatística & $g \mid$ & Sig. \\
\hline R1 & ,043 & 284 & $200^{\circ}$ & 992 & 284 & 118 \\
\hline
\end{tabular}

Fonte: elaborada pelos autores (2018).
Considerando o seguinte teste de hipótese:

$\mathrm{H} 0 \Rightarrow$ Distribuição da amostra = distribuição normal.

H1 $\Rightarrow$ Distribuição da amostra $\neq$ distribuição normal.

De acordo com a tabela da Figura 1, observa-se que tanto o teste de KolmogorovSmirnov quanto o teste de Shapiro-Wilk apresentam p-valor $>0,05$, sugerindo que deve ser aceita a hipótese nula e confirmar a normalidade da distribuição da variável dependente

\section{Ausência de multicolinearidade}

Figura 2 - Teste de correlações

\begin{tabular}{|c|c|c|c|c|c|c|}
\hline \multicolumn{7}{|c|}{ Correlações } \\
\hline & & R1 & finura & blaine & so3 & Rl \\
\hline \multirow[t]{5}{*}{ Correlação de Pearson } & R1 & 1,000 & 337 &,- 061 &, 765 &,- 417 \\
\hline & finura &, 337 & 1,000 &,- 043 &, 548 &, 163 \\
\hline & blaine &,- 061 &,- 043 & 1,000 &,- 009 &, 179 \\
\hline & so3 & .765 &, 548 &,- 009 & 1,000 &,- 300 \\
\hline & RI & -.417 & ,163 & .179 &,- 300 & 1,000 \\
\hline \multirow[t]{5}{*}{ Sig. (unilateral) } & R1 & &, 000 & .155 & .000 &, 000 \\
\hline & finura &, 000 & &, 236 &, 000 &, 003 \\
\hline & blaine & ,155 & .236 & & ,443 &, 001 \\
\hline & so3 &, 000 &, 000 & .443 & &, 000 \\
\hline & RI & .000 & .003 & .001 & .000 & \\
\hline \multirow[t]{5}{*}{$\mathrm{N}$} & R1 & 284 & 284 & 284 & 284 & 284 \\
\hline & finura & 284 & 284 & 284 & 284 & 284 \\
\hline & blaine & 284 & 284 & 284 & 284 & 284 \\
\hline & so3 & 284 & 284 & 284 & 284 & 284 \\
\hline & RI & 284 & 284 & 284 & 284 & 284 \\
\hline
\end{tabular}

Fonte: elaborada pelos autores (2018).

O resultado da análise de correlação (Figura 2) demonstra que não há nenhuma correlação forte entre as variáveis independentes. A maior correlação observada foi entre as variáveis índice de finura e índice de SO3, cujo Coeficiente de Pearson calculado foi de 0,548 , o que representa uma correlação de média intensidade. 
Figura 3 - Teste de colinearidade VIF

\begin{tabular}{|c|r|}
\hline \multicolumn{2}{|c|}{ Estatísticas de colinearidade } \\
\hline Tolerância & \multicolumn{1}{c|}{ VIF } \\
\hline & \\
& \\
, 572 & 1,750 \\
, 948 & 1,055 \\
, 536 & 1,865 \\
, 719 & 1,391 \\
\hline
\end{tabular}

Fonte: elaborada pelos autores (2018).

A multicolinearidade é definida como a presença de um alto grau de correlação entre as variáveis independentes. A tabela da Figura 3 representa o resultado do teste de colinearidade VIF (VarianceInflation Factor). O VIF mede a correlação da variável com todas as outras do modelo. Resultados abaixo de 5,0 indicam ausência de multicolinearidade.

Com base nas informações da Figura 3, podemos afirmar que as variáveis independentes não possuem forte correlação entre si, cumprindo-se, assim, mais um dos prérequisitos de validação do modelo.

\section{Teste de significância estatística do modelo}

Figura 4 - Análise da variância

\begin{tabular}{|c|c|c|c|c|c|c|}
\hline \multicolumn{7}{|c|}{ ANOVA $^{a}$} \\
\hline \multicolumn{2}{|c|}{ Modelo } & $\begin{array}{l}\text { Soma dos } \\
\text { Quadrados }\end{array}$ & gl & $\begin{array}{l}\text { Quadrado } \\
\text { Médio }\end{array}$ & $\mathrm{F}$ & Sig. \\
\hline \multirow[t]{3}{*}{1} & Regressão & 346,910 & 4 & 86,727 & 116,346 &, $000^{b}$ \\
\hline & Resíduo & 207,975 & 279 &, 745 & & \\
\hline & Total & 554,884 & 283 & & & \\
\hline \multicolumn{7}{|c|}{ a. Variável Dependente: R1 } \\
\hline \multicolumn{7}{|c|}{ b. Preditores: (Constante), RI, finura blaine, so3 } \\
\hline
\end{tabular}

Fonte: elaborada pelos autores (2018).

Considerando o seguinte teste de hipótese:

$\mathrm{H} 0=>$ As variáveis independentes não têm efeito sobre a variável dependente.

H1 => As variáveis independentes têm efeito sobre a variável dependente.

Observa-se que o p-valor $<0,05$, sugerindo que deve ser rejeitada a hipótese nula, demonstrando que as variáveis independentes têm algum efeito nos valores da variável dependente.

Figura 5 - Sumarização do modelo de regressão

\begin{tabular}{|c|c|c|c|c|c|c|c|c|c|c|}
\hline \multicolumn{11}{|c|}{ Sumarizaçäo do modelelo ${ }^{b}$} \\
\hline \multirow[b]{2}{*}{ | Modelo } & \multirow[b]{2}{*}{$R$} & \multirow[b]{2}{*}{ Rquadrado } & \multirow[b]{2}{*}{$\begin{array}{l}\text { Rquadrado } \\
\text { ajustado }\end{array}$} & \multirow[b]{2}{*}{$\begin{array}{l}\text { Eiro padräo } \\
\text { dla estimativa }\end{array}$} & \multicolumn{5}{|c|}{ Estatisticicas de mudanç̧a } & \multirow[b]{2}{*}{$\begin{array}{l}\text { Durbin- } \\
\text { Watson }\end{array}$} \\
\hline & & & & & $\begin{array}{l}\text { Mudança de } \\
\text { Rquadrado }\end{array}$ & Mudanģa $\mathrm{F}$ & gll & gl2 & $\begin{array}{c}\text { Sig. Wudança } \\
F\end{array}$ & \\
\hline 1 &, $791^{1}$ & 625 & 620 & 86338 & 625 & 116,346 & 4 & 279 &, 000 & $\overline{1,640}$ \\
\hline \multicolumn{11}{|c|}{ a. Preditores: (Constante), Rl, finura, blaine, 503} \\
\hline
\end{tabular}

Fonte: elaborada pelos autores (2018).

A análise do coeficiente de determinação $\mathrm{R}^{2}$ ajustado calculado foi de 0,620 , indicando que $62 \%$ dos valores podem ser explicados pelo modelo apresentado. Com base nas informações da Figura 5, pode-se afirmar que o modelo é estatisticamente significativo. Optase observar o $\mathrm{R}^{2}$ ajustado e não o $\mathrm{R}^{2}$ por se tratar de uma regressão linear múltipla. 


\section{ANÁLISE DOS RESÍDUOS}

Figura 6 - Histograma dos valores residuais

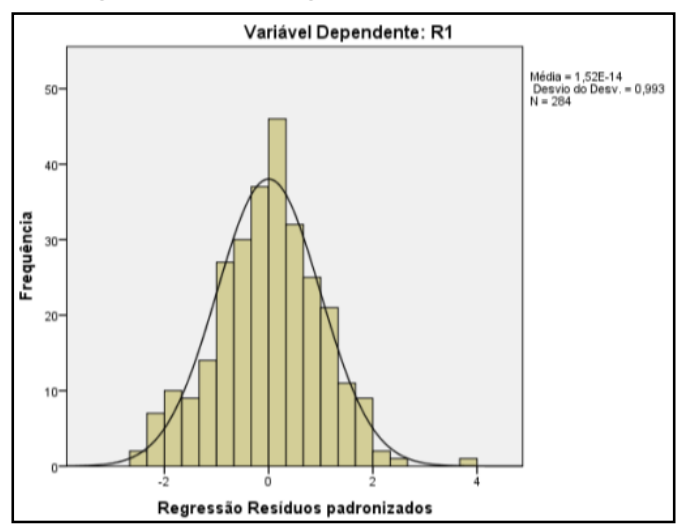

Fonte: elaborada pelos autores (2018).

Conforme o já demonstrado anteriormente, a variável dependente apresenta uma distribuição normal. Logo, pressupõe-se que os resíduos também devam ser normalmente distribuídos. Analisando o histograma da distribuição dos resíduos padronizados (Figura 6), observa-se uma curva muito próxima à distribuição normal, cumprindo-se, assim, mais um pré-requisito para validar a análise de regressão linear.

Figura 7 - Teste de Durbin-Watson

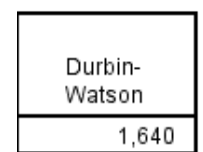

Fonte: elaborada pelos autores (2018).

O resultado do teste de Durbin-Watson indica que não há multicolinearidade entre as variáveis independentes quando o resultado se encontra na faixa de 1,5 a 2,5 . O resultado calculado pelo modelo foi de 1,640, que se coloca dentro da faixa de ausência de multicolinearidade dos resíduos.

\section{Verificação da homocedascidade}

Figura 8 - Gráfico de dispersão

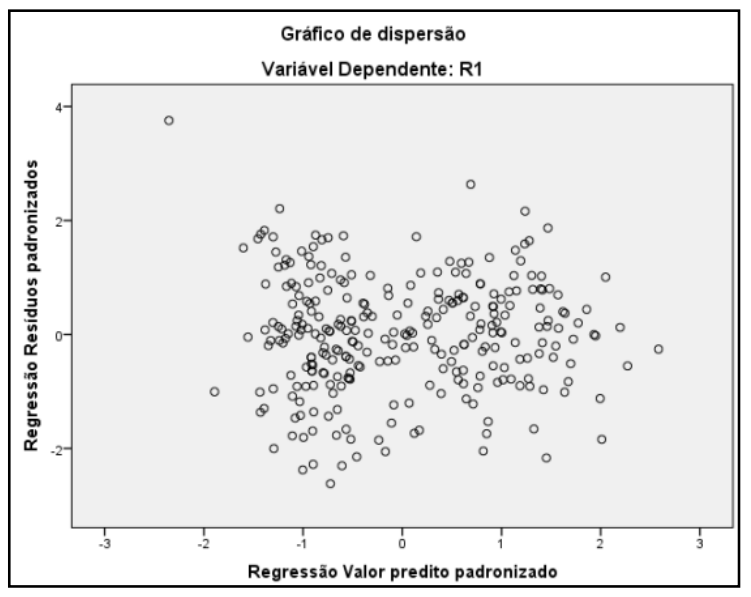

Fonte: elaborada pelos autores (2018).

A homoscedacidade pode ser verificada através da ausência de um padrão no gráfico da Figura 8. Observa-se a disposição aleatória dos pontos no gráfico dos resíduos padronizados contraposta com os valores previstos pelo modelo. O gráfico também indica uma linearidade entre as variáveis independentes e a variável dependente.

\section{Identificação de outliers nos resíduos}

Figura 9 - Estatística dos resíduos

\begin{tabular}{|c|c|c|c|c|c|}
\hline \multicolumn{6}{|c|}{ Estatísticas de resíduos $^{\text {a }}$} \\
\hline & Minimo & Máximo & Média & $\begin{array}{l}\text { Desvio } \\
\text { Padrão }\end{array}$ & $N$ \\
\hline Valor predito & 10,8580 & 16,3223 & 13,4627 & 1,10717 & 284 \\
\hline Residuo & $-2,26064$ & 3,24201 &, 00000 &, 85726 & 284 \\
\hline Valor Predito Padräo & $-2,353$ & 2,583 &, 000 & 1,000 & 284 \\
\hline Residuo Padrão & $-2,618$ & 3,755 &, 000 &, 993 & 284 \\
\hline
\end{tabular}

Fonte: elaborada pelos autores (2018).

A tabela da Figura 9 demonstra que há presença de outliers no resíduo padrão quando se observa o limite máximo. Para que os resíduos sejam considerados livres de outliers, espera-se um resultado entre -3 e +3 . O resíduo padrão apresentou valores máximos de 3,755 desvio padrão. 


\section{RESULTADOS}

Foi utilizada a regressão linear múltipla para verificar se as variáveis independentes índice de finura, blaine, índice de SO3 e índice de resíduo insolúvel (RI) são capazes de prever os valores alcançados de resistência à compressão a um dia (R1).

A análise resultou em um modelo estatisticamente significativo com valor de:

$\left[F(4,279)=116,346 ; p<0,001 ; R^{2}\right.$ Ajustado $=0,620]$

Figura 10 - Sumarização do modelo de regressão

\begin{tabular}{|c|c|c|c|c|c|c|c|c|c|c|}
\hline \multicolumn{11}{|c|}{ Sumarizaçäo do modele ${ }^{b}$} \\
\hline \multirow[b]{2}{*}{ Modelo } & \multirow[b]{2}{*}{$R$} & \multirow[b]{2}{*}{ Rquadrado } & \multirow[b]{2}{*}{$\begin{array}{l}\text { R quadrado } \\
\text { ajustado }\end{array}$} & \multirow[b]{2}{*}{$\begin{array}{l}\text { Erro padräo } \\
\text { da estimativa }\end{array}$} & \multicolumn{5}{|c|}{ Estatisticas de mudança } & \multirow[b]{2}{*}{$\begin{array}{l}\text { Durbin- } \\
\text { Watson }\end{array}$} \\
\hline & & & & & $\begin{array}{l}\text { Mudança de } \\
\text { R quadrado }\end{array}$ & Mudança F & $g \mid 1$ & $\mathrm{gl} 2$ & $\begin{array}{c}\text { Sig. Mudança } \\
F\end{array}$ & \\
\hline 1 &, $791^{\mathrm{a}}$ & .625 & .620 & 86338 & .625 & 116,346 & 4 & 279 &, 000 & 1,640 \\
\hline
\end{tabular}

Fonte: elaborada pelos autores (2018).

A análise dos coeficientes encontrados permite identificar quais variáveis possuem efeito significativo no modelo. Para tanto, é necessário aplicar o teste de hipótese de significância dos coeficientes apresentados na tabela a seguir (Figura 11), considerando que:

$\mathrm{H} 0 \Rightarrow$ A variável independente não possui efeito sobre o resultado da variável dependente $(\beta=0)$.

$\mathrm{H} 1$ => A variável independente possui efeito sobre o resultado da variável dependente $(\beta \neq 0)$.

Figura 11 - Coeficientes do modelo

\begin{tabular}{|c|c|c|c|c|c|c|c|c|}
\hline \multicolumn{9}{|c|}{ Coeficientes $^{\mathrm{a}}$} \\
\hline \multirow{2}{*}{\multicolumn{2}{|c|}{ Modelo }} & \multicolumn{2}{|c|}{ Coeficientes nåo padronizados } & \multirow{2}{*}{$\begin{array}{c}\begin{array}{c}\text { Coeficientes } \\
\text { padronizados }\end{array} \\
\text { Beta } \\
\end{array}$} & \multirow[b]{2}{*}{$t$} & \multirow[b]{2}{*}{ Sig. } & \multicolumn{2}{|c|}{ Estatísticas de colinearidade } \\
\hline & & $B$ & Erro Padrão & & & & Tolerância & VIF \\
\hline 1 & (Constante) & 8,135 & 2,127 & & 3,824 &, 000 & & \\
\hline & finura &,- 089 &, 152 & -028 & -582 &, 561 &, 572 & 1,750 \\
\hline & blaine &, 000 &, 000 & -021 &,- 565 &, 572 & 948 & 1,055 \\
\hline & so3 & 5,485 & 380 &, 723 & 14,444 &, 000 & .536 & 1,865 \\
\hline & $\mathrm{RI}$ & -158 &, 036 & -192 & $-4,442$ &, 000 &, 719 & 1,391 \\
\hline
\end{tabular}

Fonte: elaborada pelos autores (2018).

Observando-se os resultados na tabela da Figura 11, observa-se que as variáveis independentes índice de finura e blaine possuem p-valor $>0,05$, o que indica que deve ser aceita a hipótese nula de que tais variáveis não têm efeito significativo na variável dependente (R1) e podem ser descartadas do modelo.
Por outro lado, as variáveis independentes índice de $\mathrm{SO3}$ (SO3) e índice de resíduo insolúvel (RI) possuem p-valor < 0,05 , indicando que deve se rejeitar a hipótese nula. Essas variáveis, portanto, possuem efeito sobre o resultado de resistência à compressão a um dia. 
O resultado apresentado permite a demonstração da equação base da regressão para previsão dos resultados no modelo: $\mathrm{Y}=$ bo $+\mathrm{b} 1 . \mathrm{x} 1+\mathrm{b} 2 . \mathrm{x} 2$

R1

$=8,135+5,485 x_{1}$ (índice de SO3)

$-1,58 x_{2}$ (índice de resíduo insolúvel)

\section{CONSIDERAÇÕES FINAIS}

O cimento é um produto básico, insustentável e não reutilizado, utilizado principalmente como material de construção básica, aplicado em construção civil e engenharias afins. $\mathrm{O}$ cimento é um recurso fundamental que apresenta um impacto significativo no desenvolvimento da economia do país. (ZHANG; LIN, 2018)

Diante de tal importância no âmbito da economia, é indispensável a avaliação da qualidade desse produto. No entanto, por meio de amostras da qualidade, este trabalho permitiu identificar através da técnica de regressão linear múltipla, que das quatro variáveis independentes, apenas duas (índice de SO3 e índice de resíduo insolúvel) exercem alguma influência na variável dependente (resistência à compressão em um dia). As outras duas variáveis independentes analisadas (índice de finura e blaine) se mostraram insignificantes, pois essas variáveis não influenciam no resultado da variável dependente, podendo ser descartadas do modelo.

Como proposta de análise complementar, recomenda-se a repetição da técnica utilizada neste trabalho considerando-se apenas as duas variáveis independentes que se mostraram significantes para o modelo: (índice de $\mathrm{SO} 3$ e índice de resíduo insolúvel).

\section{REFERÊNCIAS}

ABCP. ASSOCIAÇÃO BRASILEIRA DE CIMENTO PORTLAND. Programa Setorial da Qualidade de Cimento Portland. 2015. Disponível em: $<$ http://pbqph.cidades.gov.br/download.ph p>. Acesso em: 08 abr., 2019.
ANDRADE, M. M. Introdução à metodologia do trabalho científico. São Paulo: Atlas 2010 .

GALLO, A. A refresher on regression analysis. Harvard Business Review, 2015.

GOUVÊA, M. A; PREARO, L. C.; DO CARMO ROMEIRO, M. Avaliação da adequação de aplicação de técnicas multivariadas em estudos do comportamento do consumidor em teses e dissertações de duas instituições de ensino superior. Revista de Administração, v. 47, n. 2, p. 338-355, 2012.

GIL, A. C. Como elaborar projetos de pesquisa. São Paulo: Atlas, 2010.

HAIR JR, J. F. et al. Análise multivariada de dados. Porto Alegre, Bookman, 2009.

HINES, W. Probabilidade e estatística na engenharia. Rio de Janeiro: LTC, 2006.

FÁVERO, L. P. et al. Análise de dados: modelagem multivariada para tomada de decisão. São Paulo: Campus, 2008.

MICHEL, M. H. Metodologia e pesquisa científica em ciências sociais: um guia prático para acompanhamento da disciplina e elaboração de trabalhos monográficos. São Paulo: Atlas, 2015.

MINAYO, M. C. S.; GOMES, S. F. D. R. Pesquisa Social: teoria, método e criatividade. Petrópolis: Vozes, 2012.

PALADINI, E. P. Avaliação estratégica da qualidade. São Paulo: Editora Atlas, 2011.

PEROVANO, D. G. Manual de Metodologia Científica para a segurança pública e defesa social. Curitiba: Editora Juruá, 2014.

ZHANG, Z.; LIN, B. Energy Conservation and Emission Reduction of Chinese Cement 
Industry: From a Perspective of Factor

Substitutions. Emerging Markets

Financeand Trade, 55(5), 967-979, 2018.

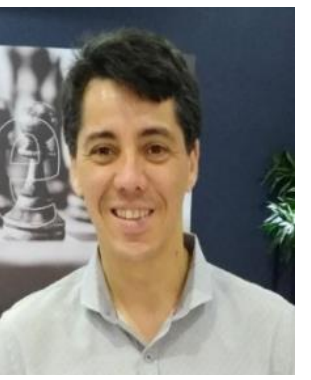

\section{DADOS BIOGRÁFICOS DOS AUTORES}

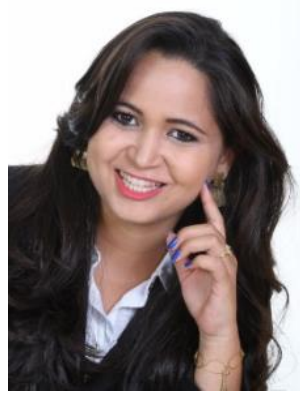

Guilherme Maranhão Barreto Pereira - Graduação em Engenharia de Produção pela Universidade do Sul de Santa Catarina (2010). Pós-graduado em Engenharia de Produção Enxuta pela PUC/PR. Atualmente é Supervisor de Produção da Votorantim Cimentos. Tem experiência na área de Engenharia Civil, com ênfase em Construção Civil.

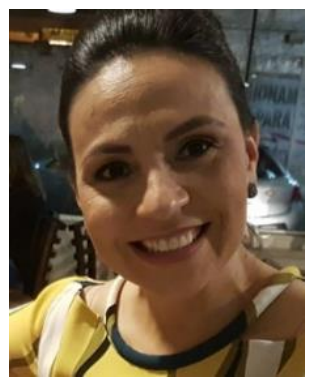

Lorena Bittencourt Bastos - Mestranda em Engenharia de Produção e Sistemas pela Universidade Federal de Santa Catarina - UFSC (2018-2019), bolsista do Conselho Nacional de Desenvolvimento Científico e Tecnológico (CNPq). Graduada em Engenharia de Produção pela Universidade de Fortaleza - UNIFOR (2016). Experiências nas áreas de PCP (Planejamento e Controle de Produção), cronoanálise e otimização de processos. Já exerceu a função de Analista de Produção e Analista de PCP em grandes empresas. Com as seguintes responsabilidades agregadas: gestão de estoque, estudo de tempos e movimentos (cronoanalista), gerenciamento de equipe, realização de indicadores de produção, manutenção preventiva de equipamentos/maquinários, desenvolvimento de um sistema Kanban, entre outros.

Karine Gonçalves - Pós-graduada em Engenharia de Produção Enxuta - Lean Manufacuting - Pontifícia Universidade católica do Paraná - PUC-PR (2017). MBA Executivo em Finanças Ibmec RJ (2009). Graduação em Ciências EconômicasUniversidade de Fortaleza - UNIFOR (2003). Consultora de Empresas no Instituto SENAI de Tecnologia em Logística de Produção - Itajaí/SC. Atua como consultora na área de Lean Manufacturing apoiando empresas de diversos setores a realizarem a transformação Lean.

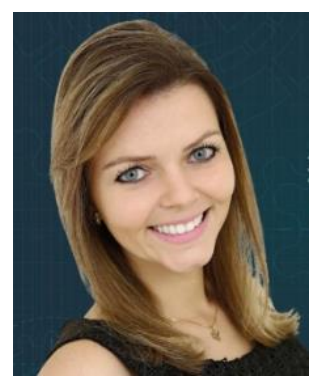

Gabriela da Rosa Witeck - Doutoranda em Engenharia Industrial pela Universidade de Minho (2019). Mestra em Engenharia de Processos pela Universidade da Região de Joinville (2018). Bolsista PIBPG (2016-2018) e atuante na linha de pesquisa em gestão da inovação tecnológica. Bacharela em Engenharia de Produção e Sistemas pela Universidade do Estado de Santa Catarina (2015). Bolsista em iniciação científica pelo CNPQ (2010-2011) no Projeto de Pesquisa intitulado: Gestão da qualidade e produtividade. Além disso, possui quatro anos de experiência na indústria multinacional automotiva (BMW do Brasil), na área de Engenharia de Produção e Engenharia de Logística. 


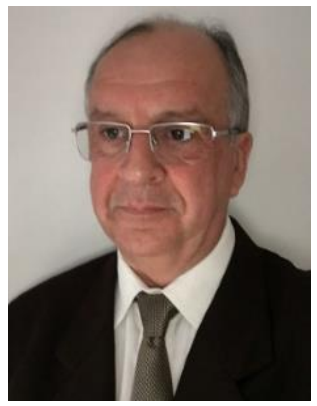

Edson Pacheco Paladini - Professor Titular do Departamento de Engenharia de Produção e Sistemas da Universidade Federal de Santa Catarina. Membro Titular da Academia Brasileira da Qualidade. Doutor em Engenharia de Produção (UFSC, 1992), Mestre em Engenharia de Sistemas (UFPb - Campina Grande, 1979), Especialização em Administração da Produção (UFPb, 1981) e Graduação em Matemática (UFSC, 1975). Atua nas áreas de Engenharia, Gestão e Avaliação da Qualidade.É autor de diversos livros sobre temas inseridos em suas áreas de atuação e de artigos técnicos publicados em periódicos qualificados no país e no exterior. Integrou o Comitê de Avaliação dos Programas de Pós-graduação da CAPES na área das Engenharias III. Coordenador do Programa de Pós-graduação em Engenharia de Produção da UFSC (2002-2006). Autor dos textos: Gestão Estratégica da Qualidade (duas edições); Gestão da Qualidade: Teoria e Prática (sete edições); Avaliação Estratégica da Qualidade (duas edições); Qualidade Total na Prática (duas edições) entre outros. 